\title{
Spin polarization in organic semiconductors
}

\author{
Yilin $\mathrm{Mi}^{\mathrm{a}}$ \\ College of Science, North China University of Technology, Beijing, China
}

\begin{abstract}
After spin was injected from ferromagnet to organic-semiconductors, spin polarization in organic semiconductors was studied theoretically considering spin-dependent conductivity in the organic-semiconductors. Our work shows that the spin injection efficiency is directly dependent on the difference between the conductivity of the spin-up and the spin-down electrons in the spin-injected organic system. Furthermore, the polaron proportion has great influence on the spin injection efficiency in the heterojunction.
\end{abstract}

Keywords: spin-dependent conductivity, spin injection efficiency, spin polarization.

\section{Introduction}

In recent years, inorganic semiconductors have attracked increasing atttentions in spin injection and detection using inorganic semiconductors [1-4]. Because organic semiconductors have hyperfine interaction, weak spin-orbit coupling, the materials are presumed to be the replacement for the inorganic semiconductors in spin transport [5-7]. Much research has been done on organic semiconductors, theoretically [8-10]. They assumed that the carriers in the organic semiconductors (OSEs) have spin 1/2 just as those in the inorganic semiconductors and studied the injected charge and spin current densities near the injecting contact. They assumed that the injected polarized electrons would be converted into polarons and bipolarons, coexisting with a given proportion $\gamma=n_{p} /\left(n_{p}+n_{b p}\right)$ in the organic polymer layer where $n_{p}$ denotes the density of polarons and $n_{b p}$ the density of bipolarons in the OSE. When spin injection stars from the ferromagnet (FM) into the OSEs, the OSEs could be polarized by this polarized injection. If the conductivity induced by the carriers with the different spin is directly proportional to their density, the conductivity induced by the spin-up and the spin-down electrons respectively are not equal any more, which depends on the parameter $\beta_{1}=\left(\sigma_{\mathrm{p} \uparrow}-\sigma_{\mathrm{p} \downarrow}\right) / \sigma . \sigma_{\mathrm{p} \uparrow, \downarrow}$ denotes the conductivity induced by the spin-up and the spin-down electrons respectively, and $\sigma$ denotes the total conductivity in the OSEs. However up to now the effect of the different density between the spin-up and the spin-down electrons on the spin injection in the spin-polarized OSEs has not emerged in the literature. In this paper, we consider spin-dependent conductivity in the polarized organic-semiconductors with infinite layers.

\section{Model}

FM/ OSEs 1D systems with semi-finite layers are considered, just as shown in Fig.1. The conductivity difference between the spin-up and the spin-down electrons induced by the spin polarization of the OSEs

${ }^{a}$ Corresponding author: miyilin@ncut.edu.cn 
was taken into account, which was induced by the spin-polarized current injection from $\mathrm{FM}(\mathrm{x}<0)$ into the OSEs layers $(x>0)$. Here we also assume that polarons and bipolarons coexist with a given proportion $\gamma$ in the OSEs layer just as in Ref. [10-11]. In the spin transport, the continuity equations for the charge and spin currents in a steady state are [12-14].

$$
\begin{gathered}
\nabla^{2}\left(\mu_{\uparrow}-\mu_{\downarrow}\right)=\lambda^{-2}\left(\mu_{\uparrow}-\mu_{\downarrow}\right), \\
\nabla^{2}\left(\mu_{\uparrow} \sigma_{\uparrow}+\mu_{\downarrow} \sigma_{\downarrow}\right)=0
\end{gathered}
$$

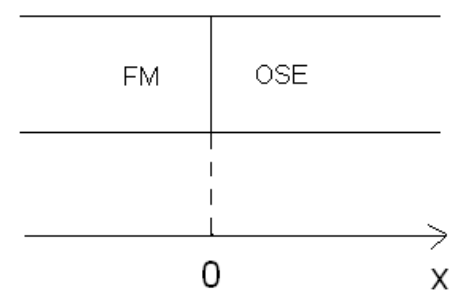

Figure 1. Spin injection device(FM/OSE) with semi-infinite layers.

Here $\mu_{\mathrm{s}}(\mathrm{s}=\uparrow, \downarrow)$ represents the electrochemical potential (ECP) for spin polarized electrons in the FM and polarons in the organic semiconductor layer.

And the basic equations related to the spin-dependent electrochemical potentials and current densities read

$$
j_{s}=\left(\sigma_{s} / e\right) d \mu_{s} / d x, j_{b p}=\left(\sigma_{b p} / 2 e\right) d \mu_{b p} / d x
$$

Where $\sigma_{\mathrm{s}}(\mathrm{s}=\uparrow, \downarrow)$ and $\sigma_{\mathrm{bp}}$ are the conductivity of spin-polarized carriers and bipolarons, $\sigma_{\mathrm{bp}}$ is the ECP of bipolarons, $\lambda$ is the spin-diffusion length in the corresponding layer, respectively. For the semi-infinite system, the solutions for Eqs. (1) in regions1 (FM), and 2(OSE) are as follows [11],

$$
\mu_{F \uparrow}-\mu_{F \downarrow}=A e^{x / \lambda_{F M}}, \mu_{p \uparrow}-\mu_{p \downarrow}=B e^{-x / \lambda p}
$$

We suppose that the conductivity of polarons and bipolarons in the OSEs layer before spin injection are as follow

$$
\sigma_{p \uparrow}=\sigma_{p \downarrow}=\frac{1}{2} \gamma \sigma, \sigma_{b p}=(1-\gamma) \sigma
$$

where $\sigma$ denotes the total conductivity of carriers in the OSE layer.

$\beta_{1}=\left(\sigma_{\mathrm{p}} \uparrow-\sigma_{\mathrm{p}} \downarrow\right) / \sigma$ is used to reflect the conductivity difference between the spin-up and the spin-down electrons induced by the spin polarization of the OSEs, when the spin is injected into the OSEs layer, the conductivity of polarons and bipolarons should be written as

$$
\sigma_{p \uparrow}=\frac{1}{2}\left(\gamma+\beta_{1}\right) \sigma, \sigma_{p \downarrow}=\frac{1}{2}\left(\gamma-\beta_{1}\right) \sigma, \sigma_{b p}=(1-\gamma) \sigma
$$

If the interfacial resistance is included at the FM/ OSEs interface, , the current is

$$
j=\sum_{s} G_{s}\left(\Delta \mu_{s} / e\right)+G_{b p}\left(\mu_{b p} /(2 e)\right)
$$

where $\mathrm{G}_{\mathrm{s}}$ and $\mathrm{G}_{\mathrm{bp}}$ denote the interfacial conductance of polarons and bipolarons, respectively. 
If in both layers including the contact the current spin polarization is introduced as

$$
\alpha(x)=\frac{j_{\uparrow}-j_{\downarrow}}{j}
$$

according to the conservation of the total current we obtain the current spin polarization of the structure,

$$
\begin{gathered}
\alpha_{0}=\frac{\gamma \beta_{0} \sigma \lambda_{F}}{\lambda_{p} \sigma_{F}} \frac{1+\frac{\sigma_{F}\left(1-\beta_{0}^{2}\right)\left(\frac{1}{G_{\downarrow}}-\frac{1}{G_{\uparrow}}\right)}{4 \lambda_{F} \beta_{0}}+\frac{\lambda_{p} \beta_{1} \sigma_{F}\left(1-\beta_{0}^{2}\right)}{\sigma \lambda_{F} \beta_{0}\left(\gamma-\beta_{1}\right)}}{\left(1-\beta_{0}^{2}\right)\left(\frac{1}{G_{\uparrow}}+\frac{1}{G_{\downarrow}}\right) \gamma \sigma}+\frac{\gamma \sigma \lambda_{F}}{4 \lambda_{p}}+\frac{\gamma\left(1-\beta_{0}^{2}\right)}{\gamma-\beta_{1}} \\
\lambda_{p} \sigma_{F}
\end{gathered}
$$

\section{Results and discussion}

Fig. 2 shows the $\sigma / \sigma_{F}$ dependence of the spin injection efficiency $\eta=\alpha_{0} / \beta_{0}$ with different $\beta_{1}$. It was found that the spin injection efficiency increases obviously with $\beta_{1}$. The solid curve expresses the result of Ref.11 where the difference between the conductivity for the spin-up and the spin-down electrons is ignored. With increasing $\beta_{1}$, the spin injection efficiency $\eta$ increases when the $\sigma / \sigma_{F}$ is fixed. Moreover, with the decreasing of the match level of conductivities $\sigma / \sigma_{F}$, the impact of $\beta_{1}$ increases. Since the conductivity of FM is usually much larger than that of the OSES materials, it seems very important to consider the impact of $\beta_{1}$ in the spin-polarized injection.

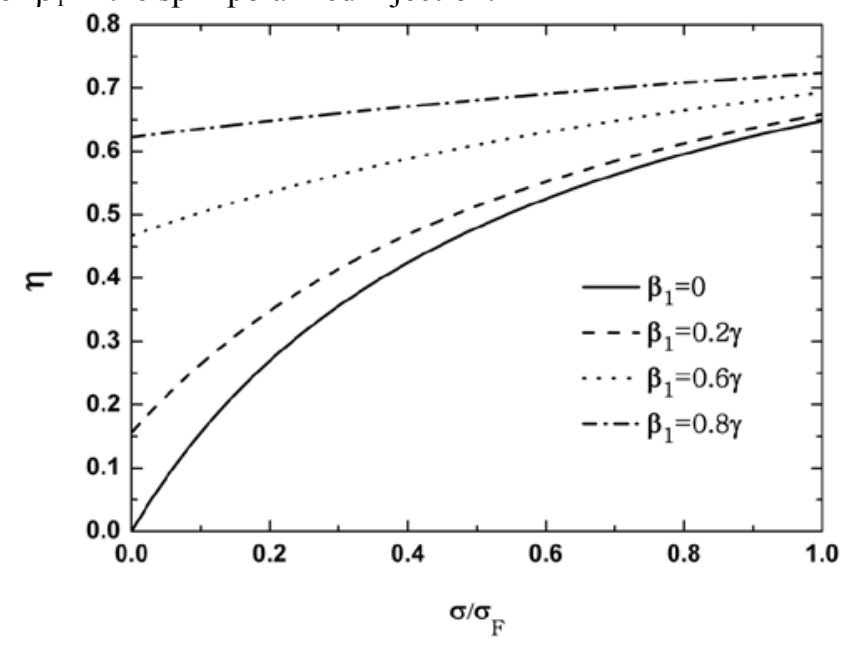

Figure 2. Dependence of the spin injection efficiency $\eta$ on the conductivity ratio $\sigma / \sigma F$ for the two materials with a transparent interface $1 / \mathrm{G} \downarrow=1 / \mathrm{G} \uparrow=0$. The solid, dot, dash-dot and dash curve are corresponding to $\beta 1=0,0.2 \gamma, 0.6 \gamma$, $0.8 \gamma$ reflecting the conductivity difference between the spin-up and the spin-down electrons in the spin-polarized OSEs respectively. Here we use parameters that are $\gamma=0.7 ; \beta 0=0.9 ; \lambda \mathrm{F}=100 \mathrm{~nm} ; \lambda \mathrm{p}=200 \mathrm{~nm}$. 


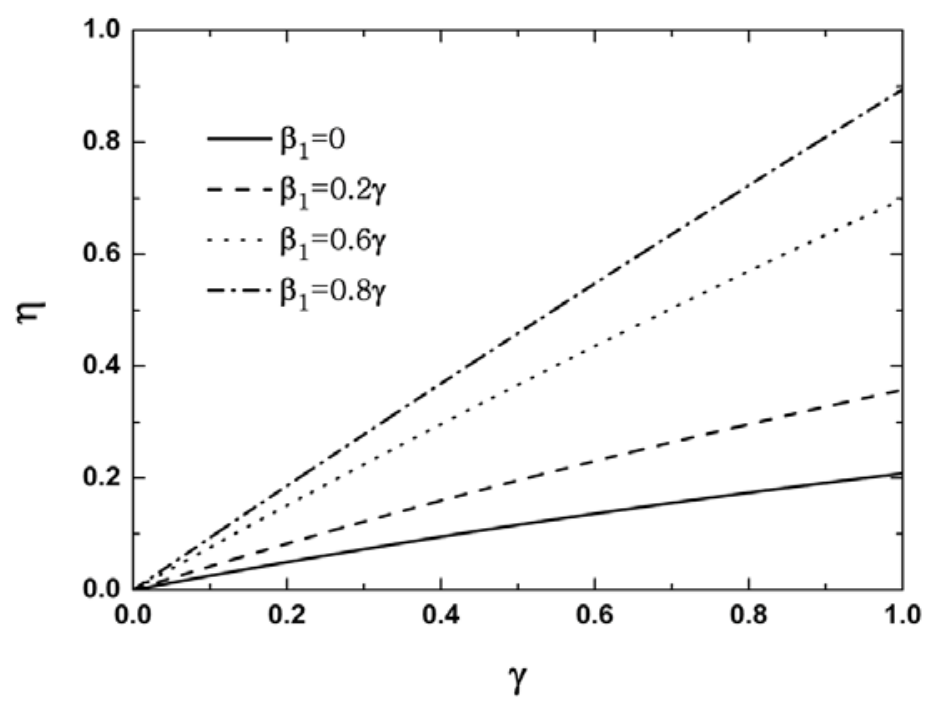

Figure 3. Dependence of the spin injection efficiency $\eta$ on the polaron proportion $\gamma$. The solid, dot, dash-dot and dash curve are corresponding to $\beta 1=0,0.2 \gamma, 0.6 \gamma, 0.8 \gamma$ reflecting the spin polarization of the OSE respectively. The bulk spin polarization of the FM layer $\beta 0$ is set to 0.9 . Other parameters are the same as in figure $3(\sigma / \sigma F=0.1 ; \lambda$ $\mathrm{F}=100 \mathrm{~nm} ; \lambda \mathrm{p}=200 \mathrm{~nm})$.

Fig. 3 shows the spin injection efficiencyi is dependent on the polaron proportion $\gamma$. With increasing the polaron proportion, the conductivity difference between the spin-up and the spin-down electrons in the spin-polarized OSEs influences the spin injection efficiency rising too. So the effect of the spin dependent conductivity on the spin injection efficiency appears so important that it can't be ignored.

\section{Conclusion}

We have obtained the effect of the spin dependent conductivity on spin transport. It is shown that the spin injection efficiency increases with increasing $\beta_{1}$ and $\gamma$. Especially, when the match level of bulk conductivities $\left(\sigma / \sigma_{\mathrm{F}}\right)$ is not very well, the effect of the spin depemdent conductivity on spin injection seems more significant.

\section{Acknowledgements}

This work is supported by the Science \& Technology Development Project of Beijing Education Committee (KM201510009011) and the National Natural Science Foundation of China (No 60576012).

\section{References}

1. Baibich M N, Broto J M, Nguyen Van Dau F, Petroff F, Etienne P, Creuzet G, Friedrich A , Chazelas J Phys. Rev.Lett. 61 (1988) 2472- 2475.

2. Moodera J, Kinder L, Wong T, Meservey R, Phys. Rev. Lett. 74 (1995) 3273-3276.

3. Wolf S A, Awschalom D D, Buhrman R A, Daughton J M, Moln'ar S V, Roukes M L, Chtchelkanova A Y, Treger D M, Science 294 (2001)1488-1495.

4. Hanbicki A T, Jonker B T, Itskos G, Kioseoglou G, Petrou A, Appl. Phys. Lett. 80 (2002) 1240-1242.

5. Dediu V, Murgia M, Matacotta F C, Taliani C, Barbanera S, Solid State Commun. 122 (2002) 181-184. 
6. Xiong Z H, Wu Di, Valy V Z, Jing S, Nature 427 (2004) 821-824.

7. Majumdar Sayani, Laiho R, Laukkanen P, Väyrynen I J, Majumdar Himadri S, Österbacka R, Appl. Phys. Lett. 89 (2006) 122114-122114.

8. Xie S J, Ahn K H, Smith K L, Bishop A R, Saxena A, Phys. Rev. B 67 (2003) 125202-125202.

9. Ruden P P, Smith D L, J. Appl. Phys. 95 (2004) 4898-4904.

10. J. F. Ren, J. Y. Fu, D. S. Liu, L. M. Mei, S. J. Xie, J. Phys.: Condens. Matter 17 (2005) 2341-2347.

11. J. F. Ren, J. Y. Fu, D. S. Liu, L. M. Mei, S. J. Xie, J. Appl. Phys. 98 (2005) 074503-074503.

12. Johnson M, Silsbee R H, Phys. Rev. Lett. 60 (1988) 377-377.

13. Alexander Khaetskii, Carlos Egues J, Daniel Loss, Charles Gould, Georg Schmidt, Laurens W. Molenkamp, Phys.Rew. B 71 (2005) 235327-235327.

14. Rashba E I, Eur. Phys. J. B 29 (2002) 513-527. 